\title{
Implementing of the offline setup correction protocol in pelvic radiotherapy: safety margins and number of images
}

\author{
Mladen Kasabašić ${ }^{1}$, Dario Faj ${ }^{1}$, Nenad Belaj ${ }^{1}$, Zlatan Faj ${ }^{2}$, Ilijan Tomaš ${ }^{1}$ \\ ${ }^{1}$ Department of Oncology and Radiotherapy, University Hospital Osijek, Croatia \\ ${ }^{2}$ Croatian Electric Power Industry, Osijek, Croatia
}

\begin{abstract}
Background. Patient positioning errors in pelvic radiotherapy at Department of Oncology and Radiotherapy in Osijek are explored in order to establish the offline setup correction protocol and determine the safety margins.
\end{abstract}

Material and methods. Film portal imaging is used during the whole treatment in order to find patient positioning errors. Eleven patients are included in the study and 420 images are analyzed. Setup errors are found by measuring distances between the center of the field and bony landmarks. Systematic and random errors are analyzed.

Results. Safety margins that should be employed at our department are $11 \mathrm{~mm}, 13 \mathrm{~mm}$ and $14 \mathrm{~mm}$ in mediolateral, craniocaudal and anteroposterior direction, respectively. Time trend is found only in an aged, obese patient with a hip problem. No action level offline setup protocol was employed by taking and averaging first four images in mediolateral and craniocaudal and 5 images in anteroposterior direction.

Conclusions. Since time trend is found only in a patient who was hard to position because of his age, obesity and the hip problem, we decided that such patients are to be positioned without a bellyboard and in supine position. Time trends are not found in all of the other patients so we employed the offline setup error protocol by averaging setup errors from the first few consecutive images. Safety margins that will ensure $90 \%$ probability of depositing at least $95 \%$ of the prescribed dose in the target are calculated. Safety margins and number of images that should be taken showed that the most inaccurate positioning was in the anteroposterior direction.

Key words: pelvic neoplasms - radiotherapy; radiotherapy dosage - methods

\section{Introduction}

Received 19 January 2007

Accepted 14 February 2007

Correspondence to: Mladen Kasabašić, Department of Oncology, University Hospital Osijek, J. Huttlera 4, 31000 Osijek, Croatia; Phone: +385 31511 478; Fax: +385 31512 219; E-mail: kasabasic.mladen@kbo.hr
The pelvic radiotherapy is often indicated for patients with cervical, uterine and rectum carcinomas. One of the problems during the radiotherapy is that total doses of 40-50 Gy to the whole pelvis can give rise to early or late complications of the 
small bowel. ${ }^{1-3}$ This is important especially because a long term survival is very often expected among those patients. The useful methods to reduce the irradiated small bowel volume are: three-field box technique, individualized normal tissue blocks, physically moved healthy tissues from the treatment field, bladder distention and prone position of the patient. , $3,4^{-}$

The use of open tabletop devices or the bellyboard, where patients are in the prone position, has been described previously. ${ }^{2-}$ ${ }^{6}$ Positioning of patients with gynecologic tumours for radiotherapy has proven to be relatively inaccurate. ${ }^{7}$ Misspositioning of the patient can give rise to complications or influence the results of the treatment. ${ }^{8}$ With now commonly available electronic portal imaging devices (EPID) it is possible to correct systematic and random field placement errors on a daily basis. ${ }^{7,9}$ The systematic error (SE) is defined as the mean displacement of the treatment isocenter from the planning isocenter, ${ }^{10}$ and the random error (RE) as a deviation of the each individual position from the mean position of the patient. The systematic error is the main factor when considering the margin size to account for setup uncertainties. ${ }^{10-12}$ When correcting the patient position, only the systematic component of the setup error must be corrected. $8,11,12$

The purpose of this study was to investigate the accuracy of daily patient positioning in our department in the bellyboard pelvis radiotherapy. Since our department is not equipped with EPID, we chose to implement strategy called no action level (NAL) protocol for reducing patient setup errors. ${ }^{13}$ It means that position of the patient will be measured during the first $N$ treatment fractions, and an unconditional correction of the setup position will be done once at the $(N$ $+1)^{\text {th }}$ fraction. In this paper we investigated how many images $(\mathrm{N})$ are needed to be performed, before deciding that the error is sys- tematic and that repositioning of the patient should be done. Too low number of images taken means that random errors (a mistake in positioning of the patient, a wrong field size, a wrong distance between the film and the patient, wrong readings of parameters on the film, wrong calculation of deviations, moving the patient during the treatment or some other reason) can cause an action and too high number of images would prolong the treatment time unnecessarily.

\section{Materials and methods}

Six patients with cancer of corporis uteri, four patients with cancer of cervicis uteri and one patient with rectal carcinoma were included in this study. The median age of the 11 patients was 64 years (47-76 years). All patients were treated using the threefield box technique at the linear accelerator Siemens Mevatron MD2. Patients are simulated at the conventional simulator SIMVIEW 3000. Shielding was done with conformal Cerrobend blocks made individually for each patient. Seven patients received the total dose of 50 Gy in 25 daily fractions, two patients received 40 Gy in 20 daily fractions followed by brachytherapy, and the patient with rectal carcinoma received the total dose of $45 \mathrm{~Gy}$ in 25 daily fractions of $1.8 \mathrm{~Gy}+$ boost $3 \times 1.8 \mathrm{~Gy}$. One patient was predicted to receive $50 \mathrm{~Gy}$ in 25 daily fractions, but she died after the $21^{\text {st }}$ fraction.

All patients were simulated in the prone position using our custom-made bellyboard. We constructed two identical bellyboards (one for the simulator, and the other for the linear accelerator) with a thickness of $8 \mathrm{~cm}$, overall size $56 \mathrm{~cm} \times 104 \mathrm{~cm}$ and opening $28.5 \mathrm{~cm} \times 30.5 \mathrm{~cm}$. Bellyboards do not have small caudal aperture for the pubic bone and they are used in the combination with the leg support. 
To reduce the volume of the small bowel within the treatment fields, patients were set in the way that the caudal border of the bellyboard opening is at the lower end of the sacroiliac joints. It means that the symphysis is out of the bellyboard opening for at least $5 \mathrm{~cm}$. The isocentar position was visualized using laser equipment and marked on patients' skin by markers. To enable the reproducible position of the patient, the opening of the bellyboard was marked by two lines on the skin.

During the simulation procedure two sets of simulation films were obtained. One film was taken for the anteroposterior field and the other for one of the lateral fields.

During the treatment session, for the verification of positioning of the patient, we were using the Kodak EC-L film system. The setup errors in patient positioning are defined by the deviations from the measured distance between the centre of the field and visible bony anatomical landmarks ${ }^{8,10}$ along the craniocaudal (CC), anteroposterior (AP), and lateral (ML) axis. Displacements of the ML and CC direction were measured from the anteroposterior field, and AP direction from the lateral field. The ML displacement was defined as a distance from the isocentar to the pelvic rim; the CC as a distance to the obturator point and the AP as a distance to the symphysis (Figure 1). We tried to measure the CC setup error also from the lateral field, but the results showed big uncertainty so we decided to omit those measurements. All deviations in the caudal direction, to the left and dorsum were marked as positive, and the deviations in the cranial direction, to the right and anterior were marked as negative.

The image acquisition was completed in $80.4 \%$. In total, 420 images were acquired for the analysis.

At the beginning of the analysis the safety margins for the setup inaccuracy were 15 $\mathrm{mm}$ in all directions.

At first, we examined the presence of time trends in any direction. The time trend is defined as drift of the field displacement in a one, systematic way during the treatment. If the time trend exists, the correction of the systematic error may not be accurate. ${ }^{11}$ Time trends were investigated using a linear regression approach and the existence of time trends was considered if the slope was greater than $4 \mathrm{~mm}$ during the treatment. This limit was used to avoid
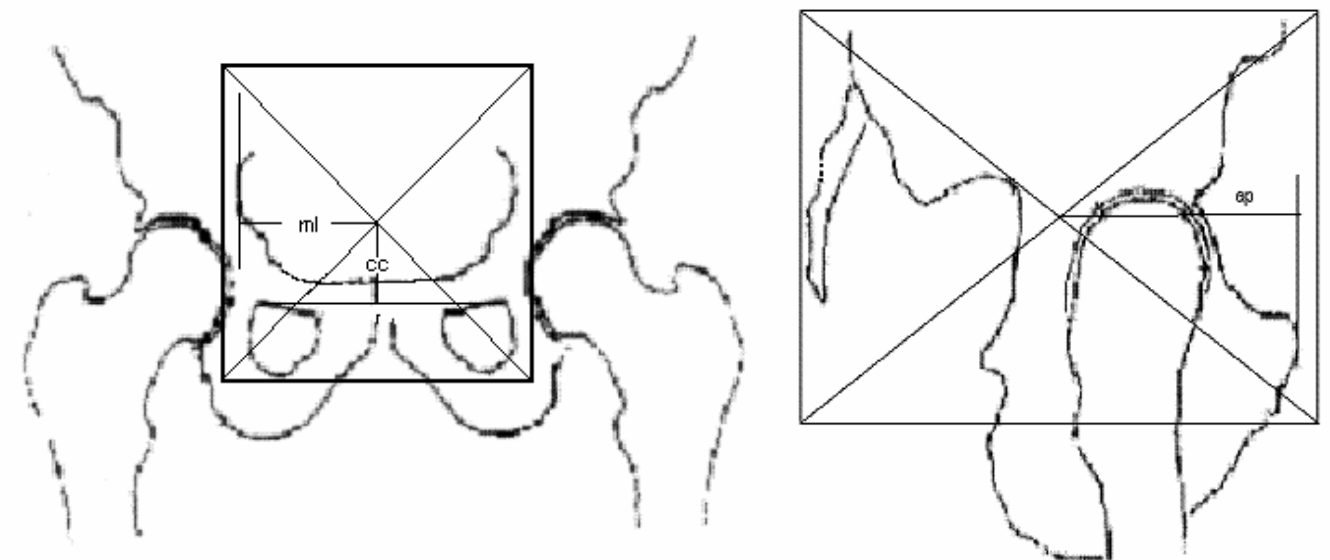

Figure 1. Determination of ML, CC and AP displacements, according to the pelvic anatomic structures, on anteroposterior and lateral projections. 


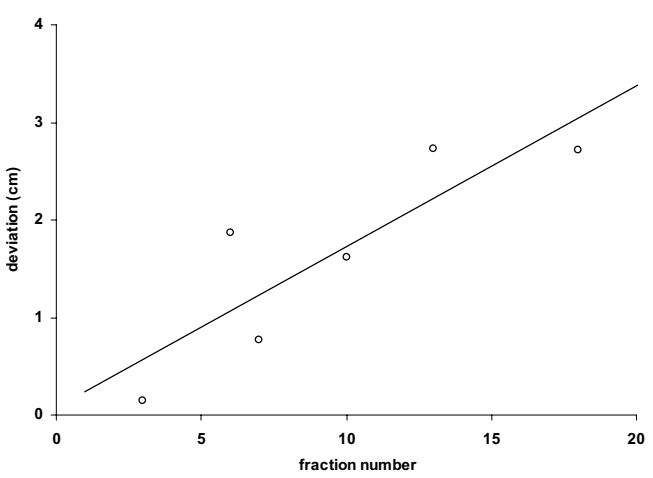

Figure 2. Presence of the time trend in the AP direction in one patient. Those data were excluded from the study.

too many patients to be excluded from the study.

The systematic error (SE) is defined as the mean displacement of the treatment isocentar from the planning isocentar, and the random error (RE) as a deviation of each individual measurement from the mean value.

The systematic error is the main factor when considering the margin size for setup uncertainties. $^{10-12}$ The systematic error for the entire group (SEeg) was defined as the arithmetic mean of all patients' systematic errors. The random deviation of the patients' SE from SEeg was estimated by 1 SD (SDse). The random deviation of all individual RE around the mean patients' RE was also estimated by 1 SD (SDre). Thus, systematic and random setup errors were calculated for the entire group of patients and the safety margin size was formed according to the sizes of those deviations.

The margin size is the one that ensures the $90 \%$ probability of depositing at least 95\% dose in the target. ${ }^{12,14}$ These values are a sensible compromise between the risk of underdosing the target volume and of excessive overdosing the surrounding healthy tissue $^{14}$. The calculation of the safety margins, $\mathrm{H}$, is done by using the following expression. $^{12}$

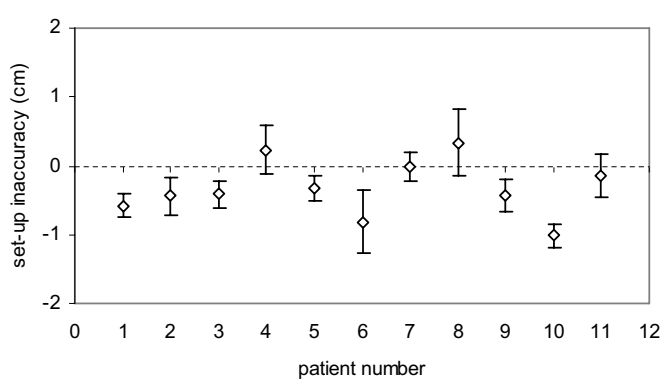

Figure 3. Mean translations and their SD for each patient in the craniocaudal direction.

$$
\mathrm{H}=2.5 \text { SDse + 0.7 SDre. }
$$

Because these margins do not include rotational errors, they should be used as the lower limit for safe radiotherapy. ${ }^{12}$

We chose to implement strategy called no action level (NAL) protocol ${ }^{13}$ for reducing patient setup errors. It means that the position of the patient will be measured during the first $N$ treatment fractions, and an unconditional correction of the setup position will be done once at the $(N+1)^{\text {th }}$ fraction. We investigated when to do the correction of systematic positioning error by evaluating setup errors during the whole treatment session.

\section{Results}

At the beginning, we checked for the presence of time trends for all patients and directions. Data were fitted as linear, and the slope of the curve is tested to be less than $4 \mathrm{~mm}$ during the whole treatment. For the ML and CC directions there was no evidence of time trends. In the AP direction, a time trend existed in an aged, obese patient with a hip problem (Figure 2).

Since that patient was very difficult to position, we excluded his AP data from our analysis.

Figures 3, 4 and 5 show the mean translations and their SDs for each patient in all three directions.

Radiol Oncol 2007; 41(1): 48-55. 


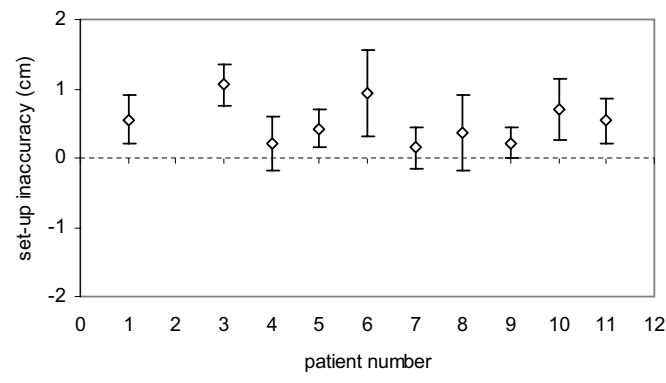

Figure 4. Mean translations and their SD for each patient in the anteroposterior direction.

The ranges of errors along the lateral, craniocaudal and anteroposterior are shown in the Table 1 together with ranges of the systematic and random components of the errors. The systematic and random errors represented by $1 \mathrm{SD}$ are also shown in the Table 1 together with the safety margins (SMs) calculated as explained before.

The calculated SMs are the lower limits for the treatment planning and we will use rounded values in upper directions. Besides, we neglected the existence of the time trends less than $4 \mathrm{~mm}$ in all directions, so this value was added to the SM sizes. Finally SMs are $11 \mathrm{~mm}, 13 \mathrm{~mm}$ and $14 \mathrm{~mm}$ in ML, CC and AP directions respectively.

To avoid random errors to cause repositioning of the patient, we investigated how many images (fractions) should be averaged

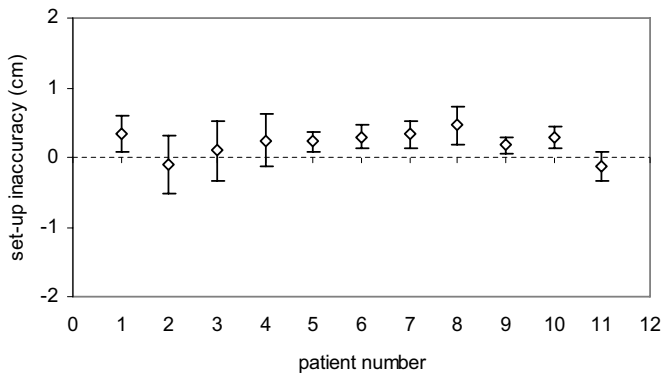

Figure 5. Mean translations and their SD for each patient in the lateral direction.

to determine whether the error is random or systematic. In a direction, the sum of all patients' REs around SE is zero. We determined the fraction number $(\mathrm{N})$ where the random error averaged over $1^{\text {th }}, 2^{\text {nd }} \ldots N^{\text {th }}$ fraction is a good approximation of the zero value. For the $j^{\text {th }}$ patient the array $n_{i, j}$ was formed by averaging all deviations of preceded fractions for a fraction i. Since REs are equally dispersed around the zero value, all patients' arrays will fast converge to the zero value. A characteristic curve for a patient is shown in Figure 6.

At a fraction $i=N$, the array value can be approximated as it reached the zero value. It means that one can decide how many fractions (N) should be averaged for a good approximation of the zero value. In this way, for the $\mathrm{j}^{\text {th }}$ patient, we approximated the systematic error at the end of the treatment

Table 1. The range of the setup errors, and systematic and random components of the setup errors, standard deviations of systematic errors (SDse), standard deviations of random errors (SDre) and calculated safety margins (SM) of 11 patients included in the study

\begin{tabular}{llll}
\hline Deviation-direction & Lateral & Craniocaudal & Anteroposterior \\
\hline Setup error-range $(\mathrm{mm})$ & -14.7 to +18 & -30.3 to +15.4 & -19.2 to +30.5 \\
Systematic error $(\mathrm{mm})$ & -1.1 to +8.9 & -11.7 to +2.4 & -1.7 to +12.1 \\
Random error $(\mathrm{mm})$ & -9.1 to 16.9 & -31.1 to 18.2 & -22.1 to 17.6 \\
SDse $(\mathrm{mm})$ & 1.9 & 2.6 & 2.5 \\
SDre $(\mathrm{mm})$ & 2.7 & 3.3 & 4.1 \\
SM $(\mathrm{mm})$ & 6.6 & 8.8 & 9.2 \\
\hline
\end{tabular}




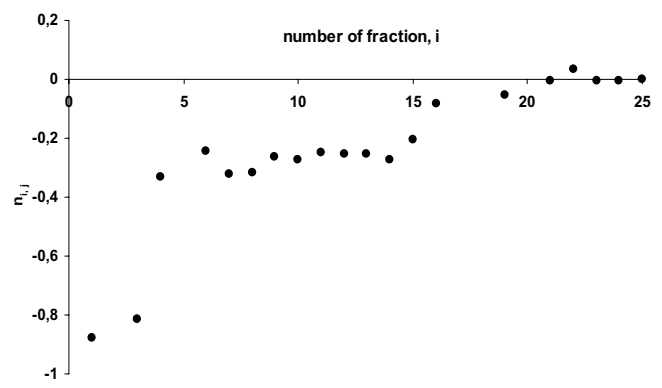

Figure 6. Array of averaged REs around SE in AP direction for a patient. After all the REs are averaged, the array ends at zero value.

$\left(\mathrm{SE}_{\mathrm{j}}\right)$, with systematic and random error at the chosen fraction. In order to be able to make this decision for a number of patients $(\mathrm{M})$, all absolute values of $\mathrm{n}_{\mathrm{i}, \mathrm{j}}$ are averaged for all of the patients. Thus, we formed the new array $k_{i}=\frac{1}{M} \sum^{M}\left|n_{i, j}\right|$ of average absolute REs at a fraction i for all fractions. Again, the array converges to the zero value and one can decide how many fractions should be averaged for a good approximation of the zero value. In this way a number of fractions, $i$ $=\mathrm{N}$, for a group of patients is found, which can be averaged to represent a good approximation of the systematic error at the end of the treatment. The calculation is done in all of the directions. Arrays of $\mathrm{k}_{\mathrm{i}}$ values are shown in Figure 7.

According to the Figure 7, numbers of images that must be taken for a patient were 3 in the ML, 4 in the CC and 5 in the AP direction. Since deviations in ML and $\mathrm{CC}$ directions are measured from the same image we decided to average four images in the ML direction as well.

\section{Discussion}

Accuracy data of daily patient positioning at our department are shown in Figures 3, 4 and 5 . The setup errors of individual meas-

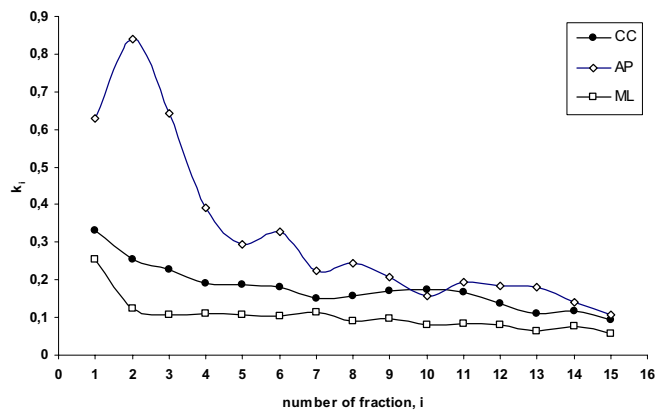

Figure 7. Averaged random deviations in CC, AP and ML directions for all of the patients during the treatment. It can be seen that random deviations are close to zero after a certain number of fractions in all of the directions and after that the slope of the curve is very mild.

urements ranged up to $18 \mathrm{~mm}, 30.3 \mathrm{~mm}$, $30.5 \mathrm{~mm}$ in ML, CC and AP directions, respectively. Out of systematic and random setup errors the safety margins were calculated. They were $11 \mathrm{~mm}$ in the ML, 13 $\mathrm{mm}$ in the CC and $14 \mathrm{~mm}$ in the AP direction. To find out how many images must be taken to decide that the setup error is systematic, the average REs of all patients during the treatment were compared. It is possible to decide when this error is close enough to zero for a group of the patients, so at that fraction, the error can be considered systematic (Figure 7). Numbers of images that must be taken are 4 in ML and $\mathrm{CC}$ directions and 5 in the AP direction. The group of the patients included in the study is assumed to be representative for treatments done at our department.

Only one patient showed time trend in one direction to be greater than $4 \mathrm{~mm}$ through the treatment (Figure 2) and those data were excluded from the study. That patient was elderly, obese and had a hip problem. We decided that the patients difficult to position by bellyboard would be planed in the supine position.

The problem that occurred during this study is non-existence of "pubic aperture" 
in our bellyboard device. This makes the position of the patient uncomfortable and they tend to move. Our measurements showed that there are no preferable directions. For future treatments the "pubic aperture" is improvised in our bellyboard.

Systematic and random errors reported are comparable to the results published in the referenced studies of gynecological patients. ${ }^{3,15}$ The safety margins extracted from this study are smaller than the margins employed before and they are on the upper side of the range of other reported results. ${ }^{14,15}$ It is important to note that the most of the published results are from advanced institutions and may not indicate variations applicable to an average, busy department. ${ }^{14}$

Although at the beginning all of the SM sizes were equally sized, the study showed that patients are moving mostly in the AP direction, so in that direction the calculated SM was the largest. This can be explained by the specific prone position of a patient and use of a bellyboard device as it was reported before. ${ }^{14,15}$ In order to apply the NAL protocol, a number of fractions for reposition value determination was found. Again, more images must be taken in AP than in CC and ML direction in order to find SE. The use of the NAL protocol means that every patient will be repositioned once during the treatment and we will explore future setup errors in order to compare them to the results of this study.

\section{References}

1. Park W, Huh SJ, Lee JE, Han Y, Shin E, Chan Y, et al. Variation of small bowel sparing with small bowel displacement system according to the physiological status of the bladder during radiotherapy for cervical cancer. Gynecol Oncol 2005; 99: 64551.

2. Ghosh K, Padilla LA, Murray KP, Downs LS, Carson LF, Dusenbery KE. Using a belly bord device to reduce the small bowel volume within pelvic radiation fields in woman with postoperatively treated cervical carcinoma. Gynecol Oncol 2001; 83: 271-75.

3. Olofsen-van Acht $\mathrm{M}$, van den Berg H, Quint S, de Boer $\mathrm{H}$, Seven M, van Somsen de Koste J, et al. Reduction of irradiated small bowel volume and accurate patient positioning by use of a bellyboard device in pelvic radiotherapy of gynecological cancer patients. Radiother Oncol 2001; 59: 87-93.

4. Hollenhorst H, Schaffer M, Romano M, Reiner M, Siefert A, Schaffer P, et al. Optimized radiation of pelvic volumes in the clinical settings by using a novel bellyboard with integrated gonadal shielding. Med Dosim 2004; 29: 173-8.

5. Huh SJ, Park W, Ju SG, Lee JE, Han Y. Small-bowel displacement system for the sparing of small bowel in three-dimensional conformal radiotherapy for cervical cancer. Clin Oncol 2004; 16: 467-73.

6. Weiss E, Richter S, Hess CF. Radiation therapy of the pelvic and paraaortic lymph nodes in cervical carcinoma: a prospective three-dimensional analysis of patient positioning and treatment technique. Radiother Oncol 2003; 68: 41-9.

7. Stroom JC, Olofsen-van Acht MJJ, Quint S, Seven M, De Hoog M, Creutzberg CL, De Boer HCJ, et al. On-line set-up corrections during radiotherapy of patients with gynecologic tumors. Int $J$ Radiat Oncol Biol Phys 2000; 46: 499-506.

8. Cazzaniga LF, Frigerio M. Errors in positioning the patient during transcutaneous radiotherapy of the pelvis. Radiol Med 1997; 94: 664-70.

9. Quint S, de Boer HCJ, van Sörnsen de Koste JR, Heijmen BJM, Olofsen van Acht MJJ. Set-up verification of cervix cancer patients treated with long treatment fields; implications of a non-rigid bony anatomy. Radiother Oncol 2001; 60: 25-9.

10. Kragelj B. Setup error and its effect on safety margin in conformal radiotherapy of the prostate. Radiol Oncol 2005; 39: 211-7. 
11. Ludbrook JS, Greer PB, Blood P, D'Yachkova Y, Coldman A, Beckham WA, et al. Correction of systematic setup errors in prostate radiation therapy: how many images to perform? Med Dosim 2005; 30: 76-84.

12. van Herk M, Remeijer P, Rasch C, Lebesque JV. The probability of correct target dosage: Dose population histograms for deriving treatment margins in radiotherapy. Int $\mathrm{J}$ Radiat Oncol Biol Phys 2000; 43: 1121-35.

13. Bortfeld T, Van Herk M, Jiang SB. When should systematic patient positioning errors in radiotherapy be corrected? Phys Med Biol 2000; 47: 297-302.

14. Booth JT. Modelling the impact of treatment uncertainties in radiotherapy. PhD thesis. Adelaide: University of Adelaide, Australia; 2002.

15. Haslam JJ, Lujan AE, Mundt AJ, Bonta DV, Roeske JC. Setup errors in patients treated with intensity-modulated whole pelvic radiation therapy for gynecological malignancies. Med Dosim 2005; 30: 36-42. 